\title{
Hybrid contracts in introductory linguistics
}

\author{
Cornelia C. Paraskevas*
}

\begin{abstract}
Wanting to shift the focus in the introductory linguistics classes in an access institution from teaching to learning, I created a tripartite (hybrid) contract that consists of three distinct tasks: labor-based tasks (collaborative forums and breakout activities); content-based tasks (assignments and final project on changing understandings about language) and self-regulated tasks (learning logs, metacognitive awareness surveys, capstone). This contract gives students agency and establishes a culture of "forgiveness" (while maintaining high standards), providing flexibility since my students' life challenges can affect their academic performance.
\end{abstract}

Keywords. contract grading; specs grading; introductory linguistics; learning logs

1. The context and the challenge. For over 30 years, I have been teaching linguistics to English majors and teacher candidates at an institution with a high admission rate (84\%), a 29\% 4-year graduation rate, and liberal admissions policies. Because Introduction to Linguistics is a required course, students view it as a 'hoop' with no obvious, immediate 'practical' application or direct connection to their other English and Education courses. Some also find the content more challenging than other courses they have to take - with the exception of math. The difficulty of the content - which frequently results in low scores - and the lack of obvious 'practical application' can result in students' loss of motivation and a let's-get-it-over-with attitude towards the material. For me, this context has created a pedagogical challenge: for the past few years, I had been looking for ways to heed Weimar's call to create learner-centered teaching. Specifically, in addition to presenting content, I wanted to help students develop their intellectual tools and learning strategies, increase their motivation and reduce their fear, give them agency, and strengthen their metacognitive awareness which is critical for learning; additionally, cognizant of my students' life challenges that can impact their academic performance, I wanted to establish a culture of “forgiveness' (while maintaining high standards) and provide some flexibility (Weimar 2003).

2. Background. To find the most appropriate solution to these challenges, I considered three different resources: contract grading, specifications grading, and learning logs. Contract grading is a practice typical in composition classrooms. Briefly, writing contracts have a set of provisions (requirements) that students must meet for a particular baseline grade (Elbow 2008). These provisions are either a combination of effort and writing quality or labor based only.

The second resource consulted was specs grading (bundling and learning targets). In bundling contracts (and assessments), each bundle consists of multiple activities, ranging from lowest to highest in cognitive outcomes. (Nilson 2015). As is the case with contract grading, students choose the grade they want to pursue and complete the appropriate bundles. Bundles that require more work, more challenging work or both allow students to higher grades. In contracts with learning targets, instructors specify the specific skills students need to meet: core (foundational) targets comprise the basic concepts in a course while advanced learning targets include more advanced discipline concepts that students may choose to master (Zuraw 2019). Students earn their target grade by demonstrating mastery of specific skills (often in multiple, successful demonstrations) through weekly quizzes, homework problems, exams, or their final project.

\footnotetext{
*Author: Cornelia C. Paraskevas, Western Oregon University (paraskc@wou.edu).
} 
The third resource consulted addressed strategies for self-learning, specifically metacognitive awareness and learning logs (Bowen 2013). In learner-centered teaching, these strategies are considered as important as content: they engender is a "deeper kind of understanding that enables [students] to retain and apply what they have learned [and makes it possible for them] to keep learning on their own for years to come" (Weimar 2003). As self-regulated learners, students become proactive in their efforts to learn, agents in control of their learning goals, able to monitor and self-reflect on their methods of learning (Zimmerman 2002).

I decided to combine elements from the three sources above and create a hybrid contract, a tripod, comprising three different categories of tasks:

- Labor-based tasks (collaborative forums and breakout activities)

- Content-based tasks (assignments and final project on changing understandings about language)

- Self-regulated tasks (learning logs, metacognitive awareness surveys, capstone).

- This contract fosters a learning environment that allows for student agency and selflearning since students choose the grade they want to pursue based on a set of requirements (Appendix). An additional, important feature of the contract are the tokens ('free passes'), the 'currency' that students can use; the number of available tokens depends on the target grade.

3. The contract in detail. Labor-based tasks (forums and breakout activities) reward student labor and curiosity while also providing points towards the students' final grade. In the forums, students collaboratively post a response to a prompt and complete a short language analysis task (for example, figuring out PS rules from a set of data). The forum posts also serve as a formative assessment tool since they give me the opportunity to determine what is clear for my students and what is 'muddy' - and in need of more detailed explanation. During the synchronous breakout activities - the second element of the labor-based component of the contract - students collaboratively engage in language analysis tasks that allow me to determine their understanding of the content. Considering the initial attitude of my students towards linguistics - they had heard it's a challenging class, technical like math and a lot of them shared that they had no "aptitude" for STEM courses - it was critical for me to provide space (and points) for them to support each other in their learning, an important consideration for students at our institution. In short, students earn points for simply engaging earnestly and honestly in the tasks - not for arriving at the correct answer. This collaborative space is truly inclusive: as students commented, "it allowed students to attempt to apply their knowledge without fear or making mistakes or messing up and allowed everyone to explore their own knowledge. It also relieves a lot of pressure and gives each student the opportunity to provide input... When a student may have struggled to grasp a concept on their own, having other students with a different perspective can help everyone gain a greater understanding." What's even more important is that this space allowed students who had completed the reading 'check' their understanding of the material as they were teaching a peer who had not completed the reading, without shaming/embarrassing them.

Content-based tasks assess (the assignments and the final project) mastery of the content they form the summative assessment for the course. The assignments students completed are typical linguistic assignments: determining the distribution and status of sounds; analyzing words into their component morphemes; showing the structure of a clause using PS rules. The final project - whereby they commented on three transformative concepts from the course - was designed to play to their strengths: students who loved the technical aspect of linguistics emphasized how important it was for them to realize that all languages have rules and patterns: instead of the 
"messiness and wishy-washiness of English," they were now discovering and formulating the patterns that governed English. "My expanded comprehension of syntax and its actual application in English has changed the way I view language."

Others were transformed by their understanding of social issues in language:

Throughout my entire education, I have been reprimanded if I did not use correct grammar, both in writing and in speech. It wasn't until we went over Dialect Variation that I began to understand what it meant for there to be no such thing as correct grammar... because there is no such thing as correct English. That completely debunked my opinions on the language statement "standard English is the same as correct English."

Understanding about language beyond the analytical methods used in the discipline was important for them: after a whole term, they realized that judgements about people should never be based on the language used: "Moving forward, I know that my changed understanding of language will influence my perspective in the future as a traveler, as a mother, as a physician, and as an advocate for change" (Allyson).

Learning logs and the metacognitive awareness survey are the cornerstone of creating intentional, expert learners since they can be used to "improve study habits, exam performance, and ultimately, new, easy and fast techniques to creating more critical thinkers" (Bowen 2013). Logs - the third leg of the contract - are included with every assignment submitted: some are 'wrappers', listing specific learning targets for the assignment and asking students to assess their confidence and ability before and after completing a task, their goals for the particular assignment and also assess the effectiveness of their preparation for completing the task. (Samples of learning logs are included in Bowen, Wirth. and Zimmerman.) The 'before' and 'after' questions are important for my students' sense of self-efficacy and provide tangible evidence of a growth mindset: "they can see that they are learning to do things they could not previously do and to treat proficiency as a skill that can be worked on" (Zuraw et al. 2019). Additionally, reflecting on their goals for each assignment and assessing their preparation encourages them to monitor and evaluate their progress (Nilson 2013).

In other logs, students revisit an earlier, graded assignment and analyze their errors, assess the source of the error and complete a similar task to the one they had difficulty with: this type of $\log$ is important as it forces them to see their mistakes as a valuable source of learning (Zimmerman).

Additionally, students fill out a metacognitive awareness survey at three points in the term (beginning, middle, and end). The survey asks them to honestly assess their study habits, learning strategies and monitoring practices. At the end of the term, the results of the surveys (together with detail from the learning logs) become part of the capstone, where students reflect on the changes in their learning practices they had observed.

Students found the metacognitive component of the contract absolutely valuable:

[they] pushed me to not settle for "being" a good student but encouraged me to continually strive for "becoming" the even better student. I will use the skills that I acquired in these logs far more frequently than I will need to construct a tree diagram or to explain the specific maxims of Grice's Cooperative Principle.

(Allyson) 
The focus on conscious learning this term made me go through trial and error processes to find strategies that worked better for me, resulting in a longer, more detailed process to completing assignments. (Alex)

These logs, in short, seem to have had the greatest impact on the students, one that will affect them throughout their college careers and beyond.

4. The difficulties and disappointments. Though in general the contract addressed the challenges I was facing, I would be remiss if I didn't mention the unexpected difficulties and disappointments. The LMS gradebook was not capable of dealing with the hybrid contract; as a result, the workload to figure out final grades was significant, consuming over 6 hours. Additional disappointment came from my students' lack of persistence: when fall term started, I was excited that almost all my students (35 out of 64) wanted to pursue an A. At the end of the term, however, only 14 students 'pushed' for the last requirement to an A. Similarly, during winter 2021, from 20 students in an Honors section who started the term aiming for an A, only 11 completed the work required for an A. Asking them informally, they told me that they hadn't managed their time appropriately and had simply run out of time to complete their last requirement for an A, the capstone reflection. To create the conditions for more As, I have carved space in the schedule so that students start working on their capstone reflection two weeks before its due date.

5. Final thoughts: The transformative power of the hybrid contract. The hybrid contract I created transformed the classroom from faculty-centered to learner- centered, with student agency at the center; each leg of the tripod contributed to my students' sense of control over their learning but also provided them equal opportunities for success for all. For instance, though students have little control over the content of the assignments completed - that is they all had to complete a phonology problem in order to demonstrate this particular learning outcome - in the final content-based task, they had significant choice in their final project, as we saw earlier. The other two legs - metacognition and labor - set conditions for success for all students: students who have had success throughout their schooling, believe that the reason for their academic achievements is their intellectual ability. While this might be partially true, we know that equally important is the ability to think about learning practices and habits: the self-regulatory activities embedded in the course gave students a sense of control over their learning and allowed all students to envision themselves as having the ability to be successful regardless of prior experiences in other classes. Finally, the labor-based leg of the tripod gave students agency but also control. Tokens proved critical for my students' success as they minimized (if not eliminated) the negative impact that life-commitments can have on academic achievement. For example, students with family obligations such as homeschooling children or helping care for family members were able to use the tokens to "buy" the extra time needed to complete their tasks for class. Similarly, students with heavy workloads (typical for my institution where 55\% of students need to work over 15 hours per week to meet expenses) were able to use the tokens to spread out the completion and submission of required work. These tokens were the cornerstone of equity in the classroom: students were not penalized for aspects of their lives over which they have little control (Nilson 2014).

In conclusion, this tripartite hybrid contract - while far from perfect - enabled me to create a learner-centered classroom, where students are given every opportunity to succeed. 


\section{References}

Bowen, Jose Antonio. 2013. Cognitive wrappers: Using metacognition and reflection to improve learning. [Web page]. https://teachingnaked.com/cognitive-wrappers-using-metacognitionand-reflection-to-improve-learning.

Elbow, Peter. 2008. A unilateral grading contract to improve learning and teaching [co-written with Jane Danielewicz]. College Composition and Communication 3. Retrieved from https://scholarworks.umass.edu/eng_faculty_pubs/3.

Inoue, Asao B. 2019. Labor-based grading contracts: Building equity and inclusion in the compassionate writing classroom. Boulder, CO: Colorado State University Open Press. https://wac.colostate.edu/books/perspectives/labor/.

Nilson, Linda. 2013. Creating self-regulated learners: Strategies to strengthen students' selfawareness and learning skills. Sterling, VA: Stylus Publishing.

Nilson, Linda. 2015. Specifications grading. Sterling, VA: Stylus Publishing.

Weimer, Maryellen. 2003. Focus on learning, transform teaching. Change 35(5). 48-54.

Wirth, Karl R. \& Dexter Perkins. 2008. Learning to learn. [Web page]. http://web.archive.org/web/20180310005012/https://www.macalester.edu/academics/geology/wirth/learn ing.pdf.

Barry J. Zimmerman (2002) Becoming a self-regulated learner: An overview. Theory Into Practice 41(2). 64-70. https://doi.org/10.1207/s15430421tip4102_2.

Zimmerman, Barry J., Adam Moylan, John Hudesman, Niesha White \& Bert Flugman. 2011. Enhancing self-reflection and mathematics achievement of at-risk urban technical college students. Psychological Test and Assessment Modeling 53(1). 141-160.

Zuraw, Kie, Ann M. Aly, Isabelle Lin \& Adam J. Royer. 2019. Gotta catch 'em all: Skills grading in undergraduate linguistics. Language 95(4). [Supplementary material]. https://doi.org/10.1353/lan.2019.0085.

WOU Shapshot. 2020. https://www.oregon.gov/highered/research/Documents/Snapshots/WOUSnapshot.pdf.

Student texts: Emily Biamont, Allyson Drury, Erica Guddat, Alex Michaels. 
Appendix

\begin{tabular}{|c|c|c|c|}
\hline Criterion & $\mathbf{A}$ & B & $\mathbf{C}$ \\
\hline Forums & 9 & 8 & 7 \\
\hline In class activities & 9 & 8 (minimum) & 7 (minimum) \\
\hline $\begin{array}{l}\text { Assignments (re- } \\
\text { quired + optional) }\end{array}$ & $\begin{array}{l}4+2 \\
\text { (combined min. } \\
90 \% \text { ) }\end{array}$ & $\begin{array}{c}4+1 \\
\text { (combined } \min .80 \%)\end{array}$ & $\begin{array}{c}4 \\
\text { (combined } \min .70 \%)\end{array}$ \\
\hline $\begin{array}{l}\text { Learning Logs/Wrap- } \\
\text { pers }\end{array}$ & 6 & 5 & No \\
\hline KAL capstone & $\checkmark$ & $\checkmark$ & No \\
\hline $\begin{array}{l}\text { Final Reflection on } \\
\operatorname{logs}\end{array}$ & $\checkmark$ & No & No \\
\hline Tokens available & 2 & 3 & 4 \\
\hline
\end{tabular}

